\title{
Bradyarrhythmia Can Increase the Plasma Level of N-Terminal Pro-Brain Natriuretic Peptide
}

Young Soo Lee, MD, PhD

Department of Cardiology, Catholic University of Daegu, College of Medicine, Daegu, Republic of Korea

Received: April 14, 2016

Revision Received: August 8, 2016

Accepted: August 16, 2016

Correspondence: Young Soo Lee, MD, PhD

Department of Cardiology, Catholic University of

Daegu, 33, Duryugongwon-ro 17beon-gil, Nam-

gu, Daegu 42472, Republic of Korea

Tel: +82-53-650-3441 Fax: +82-53-621-3166

E-mail: mdleeys@cu.ac.kr

Copyright (C) 2016 The Official Journal of Korean Heart Rhythm Society Editorial Board and MMK

Communications Limited

\section{ABSTRACT}

Background and Objectives: Myocardial wall stretch is the main trigger for pro-brain natriuretic peptide (pro-BNP) secretion. The reduced heart rate associated with bradyarrhythmia increases stroke volume, resulting in increased wall tension. Therefore, we propose that bradyarrhythmia could increase plasma N-terminal pro-BNP (NT-pro-BNP) levels.

Subjects and Methods: We enrolled 125 patients who received a temporary pacemaker because they had sinus node dysfunction (SND) or atrioventricular blocks (AVBs). Patients with renal dysfunction, hyperkalemia, reduced left ventricular systolic function (left ventricular ejection fraction [LVEF], $<40 \%$ ), and atrial fibrillation were excluded. Heart failure (HF) was defined as an NT-pro-BNP level of $>300 \mathrm{pg} / \mathrm{mL}$. We evaluated history of hypertension, diabetes mellitus, and ischemic heart disease, plasma NT-pro-BNP levels, body mass index (BMI), LVEF, left atrial diameter (LAD), and escape rhythm rate.

Results: The log plasma NT-pro-BNP level of the patients with AVBs was significantly increased compared to that of the patients with SND $(3.17 \pm 0.55$ vs. $2.93 \pm 0.64 \mathrm{pg} / \mathrm{mL}$, respectively; $p=0.03)$. The incidence of HF was $72.5 \%$ (106 patients; 44 male patients). Further, the incidence of HF was significantly higher among patients with AVBs than among patients with SND. The type of bradyarrhythmia was found to be the only predictor of HF after adjusting for age, history of hypertension, LAD, and LVEF. The LVEF, LAD, and ventricular rate were similar between the 2 groups.

Conclusion: As in the case of patients with tachyarrhythmia, bradyarrhythmia may increase plasma NT-pro-BNP levels, leading to HF. Therefore, the possibility of HF should be considered in patients with bradyarrhythmia.

Key Words: - Arrhythmias, Cardiac -Pro-Brain Natriuretic Peptide - Heart Failure 


\section{Introduction}

Brain natriuretic peptide (BNP) and $\mathrm{N}$-terminal pro-brain natriuretic peptide (NT-pro-BNP) have been used as markers for diagnosis or risk stratification in patients with heart failure (HF), stable coronary artery disease, and acute coronary syndrome. ${ }^{1}$ BNP secretion is mainly triggered by myocardial wall stretch. ${ }^{2}$ Tachycardia-induced cardiomyopathy is a well-known cause of arrhythmia-induced elevation in natriuretic peptide levels..$^{3-5}$ Therefore, we decided to investigate whether bradyarrhythmia causes an increase in plasma NT-pro-BNP levels.

\section{Subjects and Methods}

We retrospectively enrolled 125 patients who received a temporary pacemaker because they were found to have sinus node dysfunction (SND) or atrioventricular blocks (AVBs) at the emergency department of Daegu Catholic University Medical Center from March 2006 to February 2012. The SND cases included symptomatic marked sinus bradycardia $(<40 \mathrm{bpm})$ or long sinus pause (>3.0 s). AVB cases included high degree AVBs or complete AVBs. Patients with renal dysfunction, hyperkalemia, reduced left ventricular systolic function (left ventricular ejection fraction $[\mathrm{LVEF}]<40 \%$ ), and atrial fibrillation were excluded. Heart failure (HF) was defined as a NT-pro-BNP level of $>300$ $\mathrm{pg} / \mathrm{mL}$, as described in the 2012 European Society of Cardiology (ESC) Guidelines. ${ }^{6}$ We evaluated history of hypertension, diabetes mellitus, ischemic heart disease, plasma NT-pro-BNP levels, body mass index (BMI), LVEF, left atrial diameter (LAD), and ventricular rate. Blood samples were drawn from patients within 1 hour after arrival at the emergency department, and specimens were placed in 5-mL ethylenediaminetetraacetic acid tubes and immediately centrifuged. Plasma NT-pro-BNP levels were measured by using an automated system (Elecsys 2010 Roche Diagnostics, Indianapolis, IN, USA). The current study was conducted in accordance with the Declaration of Helsinki, and the Institutional Review Board of Daegu Catholic University Medical Center approved the study protocol.

\section{Statistical analysis}

The Statistical Package for the Social Sciences (SPSS) 19.0 (SPSS Inc., Armonk, NY, USA) statistical software package was used for all calculations. Data are presented as mean \pm standard deviation for continuous variables and as percentages for categorical data. The included patients were divided into 2 groups (HF versus no $\mathrm{HF}$ ) according to the measured plasma NT-proBNP levels. The unpaired Student t-test was used to analyze differences between each group. The categorical data were analyzed by using the chi-square test. The predictors of HF were analyzed by multivariate logistic regression. A p value of $<0.05$ was regarded as statistically significant.

\section{Results}

\section{Baseline characteristics}

Table 1 shows the baseline characteristics of the patients with SND $(n=53)$ and AVBs $(n=72)$. Age and BMI did not differ between the 2 groups. In addition, the levels of electrolytes such as sodium and potassium, thyroid function, and transthoracic echocardiographic parameters such as LVEF, LAD, and mitral inflow pattern determined by using Doppler were similar between SND and AVB patients. The ventricular rate was found to be slower in patients with AVBs than in those with SND, although the difference was not significant. However, NT-pro-BNP levels were significantly higher in patients with AVBs than in those with SND (Figure 1).

\section{Differences in patient characteristics according to the in-} cidence of HF

Table 2 shows the differences in patient characteristics according to the incidence of HF. A total of 166 patients (84.8\%) were diagnosed with HF. The incidence of HF was significantly higher in patients with AVBs than in those with SND (66/72; 91.7\% versus 40/53; 75.5\%, respectively; $p<0.05)$. The HF patients were significantly older and had lower BMI values than those without HF. Serum sodium levels were significantly lower in patients with $\mathrm{HF}$ as compared to those without HF. Transthoracic 
Table 1. Baseline characteristics of the patients with SND and AVB

\begin{tabular}{lccc}
\hline & SND & AVB & $P$ \\
\hline Patients No. & 53 & 72 & 0.524 \\
Age (years) & $70.8 \pm 12.3$ & $72.1 \pm 11.5$ & 0.951 \\
BMI (kg/m²) & $23.5 \pm 3.7$ & $23.5 \pm 4.4$ & 0.064 \\
Ventricular rate (beats/minute) & $43.3 \pm 11.2$ & $39.4 \pm 11.1$ & 0.181 \\
hs-CRP (mg/dL) & $10.7 \pm 12.8$ & $16.8 \pm 34.9$ & 0.030 \\
Log NT-pro-BNP* (pg/mL) & $2.93 \pm 0.64$ & $3.17 \pm 0.55$ & 0.060 \\
NT-pro-BNP (pg/mL) & $2011.4 \pm 2756.3$ & $3482.5 \pm 5737.6$ & 0.193 \\
Sodium (mg/dL) & $139.0 \pm 4.2$ & $139.9 \pm 3.7$ & 0.172 \\
Potassium (mg/dL) & $4.47 \pm 0.41$ & $4.34 \pm 0.60$ & 0.695 \\
TSH ( $(\mu \mathrm{U} / \mathrm{mL})$ & $3.39 \pm 7.86$ & $4.20 \pm 12.5$ & 0.083 \\
Free T4 (ng/dL) & $1.18 \pm 0.31$ & $1.28 \pm 0.29$ & 0.993 \\
LVEF (\%) & $59.8 \pm 9.5$ & $59.8 \pm 10.5$ & 0.624 \\
LA diameter (mm) & $41.5 \pm 7.1$ & $42.2 \pm 8.5$ & 0.308 \\
Mitral inflow E/E' & $16.2 \pm 9.5$ & $14.4 \pm 8.8$ & \\
\hline
\end{tabular}

AVB, atrioventricular block; BMI, body mass index; bpm, beats per minute; hs-CRP, high-sensitive C-reactive protein; LA, left atrium; LVEF, left ventricular ejection fraction; NT-pro-BNP, N-terminal pro-brain natriuretic protein; SND, sinus node dysfunction; TSH, thyroid stimulating hormone.

*Log NT-pro-BNP indicates the logarithmic data of NT-pro-BNP levels.

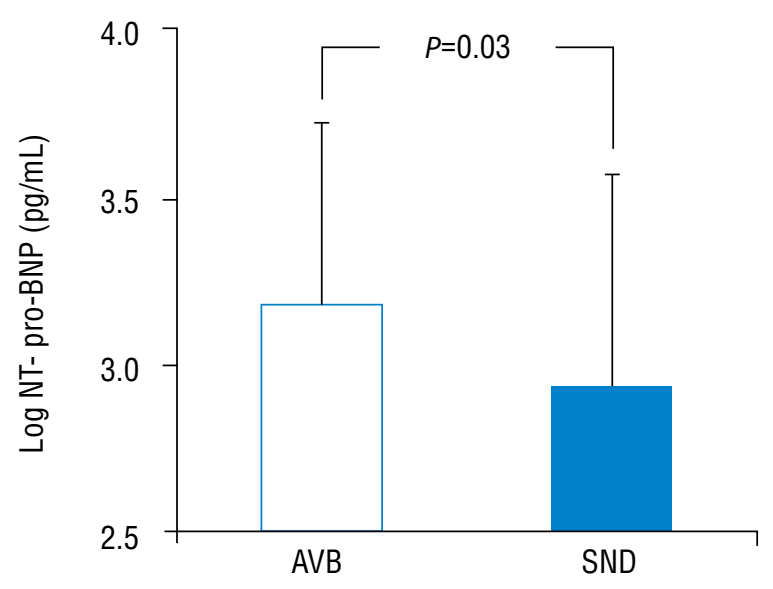

Figure 1. The level of N-terminal pro-brain natriuretic peptide (NT-pro-BNP) between atrioventricular block (AVB) and sinus node dysfunction (SND). echocardiographic parameters were similar between the patients with and without HF. Multivariate analysis revealed that the presence of $\mathrm{AVB}$ s was the only independent predictor of $\mathrm{HF}$ after adjusting for age, history of hypertension, LVEF, and LAD (Table 3).

\section{Discussion}

The present study demonstrated that bradyarrhythmia can cause an increase in plasma NT-pro-BNP levels. Furthermore, NT-pro-BNP levels were higher in patients with AVBs as compared to those with SND.

Kovats et al. reported that in elderly women with beta-blocker therapy-induced sinus arrest with a $40 \mathrm{bpm}$ junctional escape rhythm and elevated NT-pro-BNP serum levels, the stroke volume increased at the lower heart rate in order to keep up the cardiac output. Increased stroke volume results in increased wall 
Table 2. Differences in patient characteristics according to the incidence of heart failure

\begin{tabular}{|c|c|c|c|}
\hline & No $\mathrm{HF}^{*}$ & HF & $P$ \\
\hline Patient No. (\%) & $19(15.2 \%)$ & $106(84.8 \%)$ & \\
\hline Age (years) & $66.3 \pm 12.5$ & $72.5 \pm 11.5$ & 0.035 \\
\hline Male (\%) & $8(42.1 \%)$ & $44(41.5 \%)$ & 0.577 \\
\hline BMI (kg/m²) & $25.8 \pm 3.3$ & $23.0 \pm 4.1$ & 0.009 \\
\hline Hypertension (\%) & $8(42.1 \%)$ & $53(50.0 \%)$ & 0.351 \\
\hline Diabetes (\%) & $4(21.1 \%)$ & $23(21.7 \%)$ & 0.609 \\
\hline Ischemic heart disease (\%) & $2(10.5 \%)$ & $8(7.5 \%)$ & 0.469 \\
\hline Ventricular rate (beats/minutes) & $38.9 \pm 16.8$ & $41.4 \pm 10.1$ & 0.555 \\
\hline SND/AVB & $13 / 6$ & $40 / 66$ & 0.013 \\
\hline hs-CRP (mg/dL) & $4.05 \pm 2.70$ & $16.00 \pm 29.79$ & $<0.001$ \\
\hline Log NT-pro-BNP† (pg/mL) & $2.17 \pm 0.29$ & $3.23 \pm 0.49$ & $<0.001$ \\
\hline NT-pro-BNP (pg/mL) & $176.5 \pm 92.3$ & $3339.5 \pm 5011.5$ & $<0.001$ \\
\hline Sodium (mg/dL) & $141.0 \pm 2.4$ & $139.3 \pm 4.1$ & 0.014 \\
\hline Potassium (mg/dL) & $4.22 \pm 0.36$ & $4.43 \pm 0.55$ & 0.123 \\
\hline $\mathrm{TSH}(\mu \mathrm{U} / \mathrm{mL})$ & $2.80 \pm 2.13$ & $4.07 \pm 11.74$ & 0.641 \\
\hline Free T4 (ng/dL) & $1.15 \pm 0.25$ & $1.25 \pm 0.31$ & 0.188 \\
\hline $\operatorname{LVEF}(\%)$ & $61.8 \pm 6.7$ & $59.5 \pm 10.6$ & 0.356 \\
\hline LA diameter (mm) & $42.2 \pm 9.1$ & $41.9 \pm 7.7$ & 0.883 \\
\hline Mitral inflow E/E' & $13.3 \pm 10.2$ & $15.6 \pm 8.9$ & 0.318 \\
\hline
\end{tabular}

AVB, atrioventricular block; BMI, body mass index; bpm, beats per minute; HF, heart failure; hs-CRP, high-sensitive C-reactive protein; LA, left atrium; LVEF, left ventricular ejection fraction; NT-pro-BNP, N-terminal pro-brain natriuretic protein; SND, sinus node dysfunction; TSH, thyroid stimulating hormone.

*HF was defined as a patient who complained dyspnea and increased NP-pro-BNP levels (>300 pg/mL).

t Log NT-pro-BNP indicates the logarithmic data of NT-pro-BNP levels.

tension, which in turn triggers natriuretic peptide secretion. ${ }^{8}$ Further, Meiracker et al. ${ }^{9}$ demonstrated that in hypertensive patients, decreased heart rate due to beta-blocker therapy increased plasma atrial natriuretic peptide and BNP concentrations.

Pan et al. ${ }^{10}$ reported that patients with SND, AVB, and atrial fibrillation had increased plasma NT-pro-BNP levels as compared to the controls, and NT-pro-BNP levels were lower in patients with SND than in patients with AVB; this finding is consistent with our result. They also demonstrated that the severity of atrioventricular asynchrony was an independent determinant of NT-pro-BNP levels. Atrioventricular asynchrony leads to stretching of the atrial wall and increased ventricular end-diastolic pressure, resulting in increased secretion of NT-pro-BNP from the atrium and ventricle. ${ }^{11-12}$ Koch et al. ${ }^{13}$ reported that patients with dual-chamber pacing had significantly lower BNP values as compared to those with single-chamber ventricular pacing due to the loss of atrioventricular synchrony.

Taken together, bradyarrhythmia due to beta-blocker therapy, AVBs, or SND can lead to elevated plasma NT-pro-BNP levels by 
Table 3.Multivariate logistic analysis to determine the predictor of heart failure

\begin{tabular}{lccc}
\hline & Odds ratio & $95 \% \mathrm{Cl}$ & $P$ \\
\hline Age & 1.046 & $0.997,1.097$ & 0.066 \\
Hypertension & 1.602 & $0.509,5.041$ & 1.602 \\
LVEF & 0.963 & $0.910,1.019$ & 0.194 \\
LA diameter & 0.968 & $0.904,1.036$ & 0.349 \\
Type of bradyarrhythmia (AVB) & 3.915 & $1.274,12.029$ & 0.017 \\
\hline
\end{tabular}

$A V B$, atrioventricular block; $C l$, confidence interval; $L A$, left atrium; LVEF, left ventricular ejection fraction.

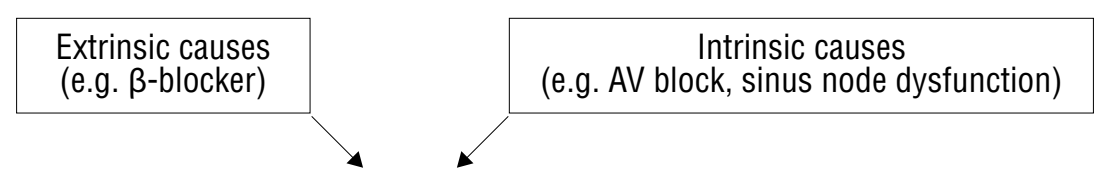

\section{Bradycardia}

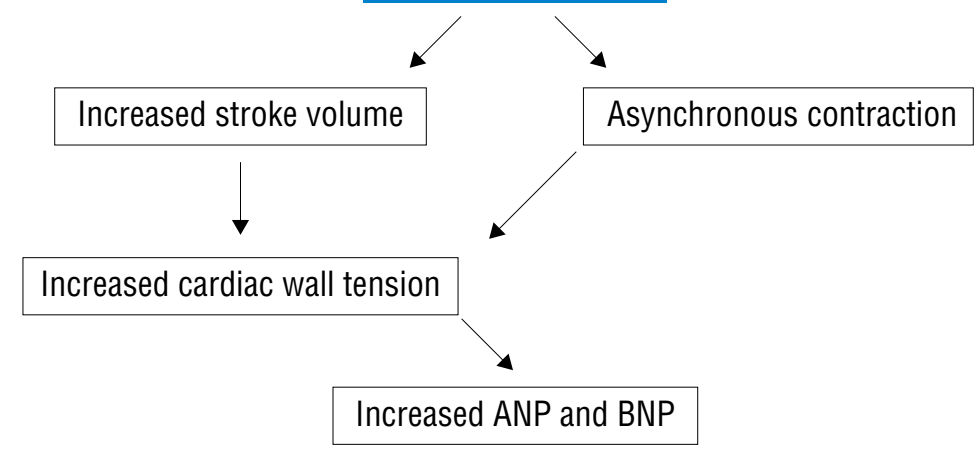

Figure 2. The mechanisms for increased plasma BNP level by bradycardia.

ANP, atrial natriuretic peptide; AV, atrioventricular; BNP, brain natriuretic peptide.

increasing cardiac wall stretch through an increase in stroke volume or loss of atrioventricular synchrony (Figure 2).

\section{Conclusion}

As in the case of patients with tachyarrhythmia, bradyarrhythmia, especially that caused by AVBs, may cause an increase in plasma NT-pro-BNP levels, leading to HF. Therefore, the possibility of HF should be considered in patients with bradyarrhythmia.

\section{References}

1) Daniels LB, Maisel AS. Natriuretic peptides. J Am Coll Cardiol. 2007;50:2357-2368.

2) Schmitt M, Cockcroft JR, Frenneaux MP. Modulation of the 
natriuretic peptide system in heart failure: from bench to bedside?

Clin Sci (Lond). 2003;105:141-160.

3) Crozier IG, Ikram H, Nicholls MG, Espiner EA, Yandle TG. Atrial natriuretic peptide in spontaneous tachycardias. Br Heart $J$. 1987;58: 96-100.

4) Jourdain P, Bellorini M, Funck F, Fulla Y, Guillard N, Loiret J, Thebault B, Sadeg N, Desnos M. Short-term effects of sinus rhythm restoration in patients with lone atrial fibrillation: a hormonal study. EurJ Heart Fail. 2002;4:263-267.

5) Kido S, Hasebe N, Ishii Y, Kikuchi K. Tachycardia-induced myocardial ischemia and diastolic dysfunction potentiate secretion of ANP, not BNP, in hypertrophic cardiomyopathy. Am J Physiol Heart Circ Physiol. 2006;290:H1064-1070.

6) McMurray JV J, Adamopoulos S, Anker SD, Auricchio A, Bo“hm M, Dickstein K, Falk V, Filippatos G, Fonseca C, Gomez-Sanchez MA, Jaarsma T, Køber L, Lip Y.H. G, Maggioni PA, Parkhomenko A, Pieske BM, Popescu BA, Rønnevik PK, Rutten FH, Schwitter J, Seferovic P, Stepinska J, Stepinska PT, Voors AA, Zannad F, Zeiher A. ESC Guidelines for the diagnosis and treatment of acute and chronic heart failure 2012. Eur Heart J. 2012;33:1787-1847.

7) Kovats T, Wettstein A, Nagy E, Tomcsanyi J. Bradycardia can induce increased serum natriuretic peptide-level. Int J Cardiol. 2008;123:e43-e44.

8) Kovats T, Tomcsanyi J. Bradycardia and B-type natriuretic peptide. Int J Cardiol. 2009;136:238-239.

9) van den Meiracker AH, Lameris TW, van de Ven LL, Boomsma F. Increased plasma concentration of natriuretic peptides by selective beta1-blocker bisoprolol. J Cardiovasc Pharmacol. 2003;42:462468.

10) Pan W, Su Y, Hu K, Shu X, Ge J. Effect of bradyarrhythmia on the plasma levels of $\mathrm{N}$-terminal pro-brain natriuretic peptide. Int $J$ Cardiol. 2009: 24;136:105-107.

11) Noll B, Krappe J, Göke B, Maisch B. Influence of pacing mode and rate on peripheral levels of atrial natriuretic peptide (ANP). Pacing Clin Electrophysiol. 1989;12:1763-1768.

12) Kürüm T, Yüksel M, Ozbay G, Söyük S, Türe M. Relationship with plasma neurohormones and dyssynchrony detected by Doppler echocardiography in patients undergoing permanent pacemaker implantation. Acta Cardiol. 2003;58:499-505.

13) Koch A, Zink S, Dittrich S. Plasma levels of B-type natriuretic peptide in children and adolescents with high degree atrioventricular block. Int J Cardiol. 2009;134:429-430. 